\title{
Impacts de l'assainissement autonome sur la qualité des eaux de puits dans la Commune de Sèmè-Podji (Sud-Bénin)
}

\author{
Flavien Edia DOVONOU ${ }^{1 *}$, Abdoukarim ALASSANE${ }^{1}$, Vidédji Naéssé ADJAHOSSOU ${ }^{2}$, \\ Bibiane AGBODO $^{1}$, Roufai DJIBRIL ${ }^{1}$ et Daouda MAMA ${ }^{1}$ \\ ${ }^{1}$ Université d'Abomey-Calavi (UAC)/Institut National de l'Eau, Laboratoire d'Hydrologie Appliquée (LHA), \\ 01 BP 526 Cotonou, Bénin. \\ ${ }^{2}$ Université Nationale des Sciences Technologies Ingénierie et Mathématiques (UNSTIM)/ FAST, Laboratoire \\ Pierre Pagney Climat, Eau, Ecosystème et Développement (LACEEDE), 01 BP 2417 Cotonou, Bénin. \\ *Auteur correspondant ; E-mail : dovflav@yahoo.fr
}

\section{RESUME}

L'accès à l'eau potable et aux installations d'assainissement hygiéniques est essentiel pour la santé. Cette étude menée à Sèmè-Podji, a permis de déterminer les facteurs responsables de la dégradation des ressources en eau. Les analyses chimiques et bactériologiques des échantillons d'eau prélevés dans les puits, ont été effectuées au Laboratoire d'Hydrologie Appliquée de l'Université d'Abomey-Calavi (Bénin). Les résultats montrent que les eaux de puits contiennent de nombreux polluants. Sur le plan chimique, de fortes teneurs en nitrite $(5 \mathrm{mg} / \mathrm{L})$, nitrate $(100 \mathrm{mg} / \mathrm{L})$, phosphates $(60 \mathrm{mg} / \mathrm{L})$ et en ammonium $(40 \mathrm{mg} / \mathrm{L})$ ont été enregistrées dans ces eaux. Au niveau microbiologique, on dénombre de nombreux coliformes totaux dans ces eaux. Cette pollution est due à la présence des latrines, de tas d'ordures et de porcheries. Différentes corrélations entre les paramètres physiques (distance puits-latrine, position topographique, nombre de latrines, position de la source de pollution, type d'aménagement du puits) et la qualité de l'eau ont été effectuées et ont permis de déterminer les différents facteurs de pollution de ces eaux. Le sens d'écoulement des eaux souterraines, les fluctuations du niveau de la nappe, la distance du point d'eau à la source de pollution, et à moindre degré l'aménagement sont les facteurs qui contrôlent la qualité des eaux de l'aquifère superficiel dans la Commune de Sèmè-Podji.

(C) 2017 International Formulae Group. All rights reserved.

Mots clés : Sèmè-Podji, eau de puits, latrines, pollution.

\section{Impacts of autonomous sanitation on the quality of wells water in the commune of Sèmè-Podji (South Benin)}

\begin{abstract}
Access to safe drinking water and sanitation is essential for health. This study conducted in SèmèPodji aimed at determines the factors responsible for the degradation of the water resources. The chemical and bacteriological analyzes of the water samples taken from the wells were carried out at the Laboratory of Applied Hydrology of Abomey-Calavi University. The results show that wells water contain many pollutants. On the chemical point of view, nitrite $(5 \mathrm{mg} / \mathrm{L})$, nitrate $(100 \mathrm{mg} / \mathrm{L})$, phosphate $(60 \mathrm{mg} / \mathrm{L})$ and ammonium $(40 \mathrm{mg} / \mathrm{L})$ were recorded in those samples. At the water microbiological level, there are many total coliforms
\end{abstract}


in these waters. Pollution is caused by the presence of latrines, piles of garbage and pigsty. Different correlations between physical parameters (sink-latrine distances, topographic position, number of latrines, position of the source of pollution, type of wells management) and water quality were carried out and allowed to determine the different factors of pollution of these waters. Groundwater flow direction, groundwater level fluctuations, distance from water point to the source of pollution and management to a lesser extent are factors that control the quality of waters from the shallow aquifer in Sèmè-Podji.

(C) 2017 International Formulae Group. All rights reserved.

Keywords: Sèmè-Podji, well water, latrines, pollution.

\section{INTRODUCTION}

L'une des conditions nécessaires pour permettre à une population de disposer de l'eau de boisson de qualité est de maintenir son cadre de vie dans un état de salubrité permanent grâce à un système adéquat de traitement d'eau et d'assainissement du milieu (OMS, 2006). L'assainissement de base, ce sont des toilettes (WC, latrines) et les systèmes qui permettent la gestion des rejets domestiques (les eaux usées de la maison et surtout les excréments). En effet, sans système d'assainissement, les excréments et les eaux usées vont directement dans la nature et contaminent les eaux que des familles entières consomment au quotidien. Par ailleurs, les latrines améliorées garantissent une séparation hygiénique des excrétas de tout contact humain évitant ainsi le transport des germes par l'Homme pouvant polluer l'eau de consommation (Agassounon et al., 2012). Outre le problème des excréta humains, l'absence de gestion des déchets solides a de l'impact sur la nappe phréatique. En effet, la dégradation des déchets produit du lixiviat, c'est à dire un jus lié notamment à la dégradation de la matière organique. En période de pluie, lorsque les conditions d'imperméabilité des sols sont insuffisantes, le lixiviat peut polluer les nappes phréatiques.

Les maladies d'origine hydrique représentent le principal problème de santé publique dans les pays en développement. L'inefficience des services d'approvisionnement en eau et d'assainissement dans certaines zones précaires est due, entre autres, à l'occupation anarchique des sols, à l'extrême pauvreté des populations et à l'insuffisance des moyens financiers de la part des pouvoirs publics.

En Afrique, 344 millions de personnes n'avaient pas accès à une source améliorée d'eau potable en 2010 et 222 millions défèquent à l'air libre (Agassounon et al., 2012). Au Bénin, les sources d'eau sont soumises à diverses menaces dues à la poussée démographique qui est une crainte pour les générations futures (Dovonou, 2012). Ainsi, 65\% des ménages ne disposent pas d'ouvrages d'assainissement adéquats et $25 \%$ n'ont pas accès à une eau potable (Agassounon et al., 2012). La campagne de sensibilisation sur le lavage des mains à l'eau et au savon aux moments critiques n'est pas encore rentrée dans les habitudes. Seulement à peine $4 \%$ des ménages en milieu rural et périurbain disposent d'équipements de lavage des mains (INSAE, 2007). Selon la même source, 60 à $70 \%$ des causes de consultations sont actuellement dues à des maladies liées à l'eau, à l'hygiène et à l'assainissement. Au début des années 80, le gouvernement du Bénin a abordé résolument la Décennie Internationale de l'Eau Potable et de l'Assainissement (DIEPA) en se fixant pour objectifs, l'amélioration de l'assainissement urbain, la dotation des écoles et des formations sanitaires en latrines, la couverture à $80 \%$ des besoins en eau des populations rurales et à $100 \%$ les besoins des populations urbaines et la prise en charge progressive de l'entretien de tous les ouvrages du secteur par leur utilisateur (Ahoudi, 2015). Dans la même optique, le Bénin a souscris en 2002, aux 
Objectifs du Millénaire pour le Développement (OMD) qui sont des engagements pour la construction d'un monde meilleur à fin 2015. L'aspiration à un cadre de vie sain est abordée dans l'objectif $n^{\circ} 7$ «Assurer un environnement durable ». En sa cible 10, cet objectif vise à «réduire de moitié, à fin 2015, le pourcentage de la population qui n'a pas accès durable à un approvisionnement en eau potable et aux ouvrages d'assainissement adéquats ».

Pour faire face à ces défis, le Bénin s'est engagé depuis 2003, dans un processus de décentralisation afin de promouvoir le développement au niveau local. Ainsi, selon la loi $\mathrm{n}^{\circ}$ 97-029 du 15 janvier 1999 portant organisation des communes en République du Bénin, les maires sont entièrement responsabilisés pour garantir un environnement sain et un accès durable à l'eau potable et aux ouvrages adéquats d'assainissement à leurs administrés. Elles devraient donc s'impliquer dans la définition des priorités en matière d'assainissement, d'amélioration de l'hygiène et de la salubrité au sein des communautés. Mais aujourd'hui, force est de constater qu'en dépit des efforts déployés par les collectivités locales pour l'offre de services aux populations dans les domaines de l'approvisionnement en eau potable et la jouissance d'un environnement sain, il reste encore beaucoup de choses à faire pour combler leurs attentes. La Commune de Sèmè-Podji n'est pas exempte de cette situation. En effet, à Sèmè-Podji, tous les ménages ne sont pas abonnés au réseau de la Société Nationale des Eaux du Bénin (SONEB). Ce qui amène certains habitants à consommer les eaux de puits. Pour ces derniers, les eaux issues de ces puits paraissent claires et sans odeur; ainsi, ils assimilent leur qualité à celle de la SONEB.

Les problèmes qui se posent aux techniciens communaux et aux décideurs chargés de l'exploitation du réseau de la SONEB se résument souvent en l'absence d'information sur la qualité de l'eau des sources alternatives notamment les puits traditionnels.

La connaissance de la qualité physico-chimique et microbiologique des eaux de puits dans cette commune mérite une attention particulière puisqu'elle constitue, d'une part, une zone où la nappe phréatique est affleurante, et d'autre part, une zone qui connaît une prolifération des ouvrages d'assainissement autonome dans un contexte où les normes d'assainissement en République du Bénin (construire la latrine à une distance minimale de 15 mètres d'un puits) ne sont toujours pas respectées.

\section{MATERIEL ET METHODES \\ Présentation de la Commune de Sèmè- Podji}

Située entre les parallèles $6^{\circ} 22^{\prime}$ et $6^{\circ} 28^{\prime}$ de latitude Nord et les méridiens $2^{\circ} 28^{\prime}$ et $2^{\circ} 43^{\prime}$ de longitude Est, la Commune de Sèmè-Podji est située dans le Département de l'Ouémé, au Sud-Est de la République du Bénin sur la côte Atlantique. Elle s'étend sur une superficie de $250 \mathrm{Km}^{2}$, soit environ $0,19 \%$ de la superficie nationale. La Commune de Sèmè-Podji est limitée au Nord par les Communes de Porto-Novo et Aguégué, au Sud par l'Océan Atlantique, à l'Est par la République Fédérale du Nigeria et à l'Ouest par la Commune de Cotonou. Sèmè-Podji est une plaine côtière encastrée dans un complexe de plans d'eau (Océan Atlantique, lagune de Porto-Novo, fleuve Ouémé et lac Nokoué). Le relief très bas varie par endroit entre 0 et $6 \mathrm{~m}$ environ d'altitude. Il est majoritairement composé de marécages, de sables fins inaptes aux activités agricoles et de plans d'eau. La superficie cultivable fait $39,5 \%$ de la superficie totale de la commune, et baigne dans un climat soudano guinéen caractérisé par deux saisons sèches (décembre à février et août à septembre) et deux saisons pluvieuses (avril à juillet et octobre à novembre). La température moyenne fait environ $27{ }^{\circ} \mathrm{C}$ avec une humidité relative élevée. L'influence du vent côtier sur le climat crée souvent des 
perturbations cycliques qui font de la Commune de Sèmè-Podji, une des zones les plus arrosées du Sud Bénin avec une moyenne pluviométrique dépassant annuellement 1100 mm. Elle compte 224207 habitants selon les résultats du quatrième Recensement Général de la Population et de l'Habitation (RGPH4) effectué en 2013.

\section{Matériel de collecte de données}

Dans le cadre de cette étude, pour la collecte des données, on a utilisé des outils contenus dans le Tableau 1.

\section{Méthodes de collecte de données Recherche documentaire}

Elle a été faite à travers la lecture de plusieurs documents scientifiques (revues, publications, rapports, mémoires, thèses) à la bibliothèque centrale l'Université d'AbomeyCalavi. De plus, les rapports d'études sur l'hygiène et l'assainissement ont été consultés au niveau du service technique de la marie de Sèmè-Podji.

\section{Enquêtes et collecte de données de terrain}

Des investigations ont été menées sur le terrain et au laboratoire. Ces travaux de terrain se sont déroulés dans les localités d'Ekpè III et Podji-Agué dans la Commune de SèmèPodji. Les analyses et les traitements des eaux ont été effectués au Laboratoire d'Hydrologie Appliquée de l'Université d'Abomey-Calavi en Octobre 2015. Les investigations développées sur le terrain ont porté essentiellement sur le recensement exhaustif et la description de tous les ouvrages alternatifs d'approvisionnement en eau : il a été question d'une part, d'identifier toutes les sources potentielles de pollution des eaux de la nappe phréatique dans les zones de prélèvement des échantillons d'eau de puits, et d'autre part, de déterminer les distances minimales qui séparent les puits des sources de pollution.

Les coordonnées géographiques de chacun des points sélectionnés ont été relevées à l'aide du récepteur GPS.
Echantillonnage et techniques de prélèvement d'eau de puits

Deux campagnes d'échantillonnages d'eaux et de mesures des paramètres physicochimiques ont été menées (une campagne en saison des pluies dans le mois d'Octobre 2015 et une autre en saison sèche dans le mois de janvier 2016). Vingt puits d'eau désignés par P1, P2, P3, P4, P5, P6, P7, P8, P9, P10, P11, P12, P13, P14, P15 P16, P17, P18, P19, et $\mathrm{P} 20$, ont été choisis à raison de dix par localité, Ekpè III et Podji-Agué, en fonction des aménagements, la situation par rapport aux latrines, l'importance pour la population, les activités menées dans leurs alentours, le consentement du propriétaire à inscrire son puits dans l'étude et la pérennité de l'ouvrage. Au total quarante échantillons d'eaux ont été prélevés au cours des deux campagnes.

De façon pratique, les échantillons d'eau ont été prélevés à l'aide des puisettes. En effet, cette disposition a été adoptée pour éviter à ce que les ménages soupçonnent de notre part, une contamination de leur puits si on utilisait d'autres outils de prélèvement.

L'eau, une fois puisée, est mise dans des bouteilles et flacons d'échantillons déjà apprêtés depuis le laboratoire pour la circonstance. Ces bouteilles et flacons sont ensuite placés dans une glacière contenant des accumulateurs de froid pour leur conservation à $4{ }^{\circ} \mathrm{C}$ et analysés immédiatement au laboratoire.

\section{Analyse au laboratoire}

Les analyses ont été faites au Laboratoire d'Hydrologie Appliquée de l'Université d'Abomey-Calavi. Elles ont concernées les paramètres ci-après: Les paramètres physico-chimiques (température, $\mathrm{pH}$, oxygène dissous, nitrates, nitrites, ammonium et phosphates) et les paramètres microbiologiques. (coliformes totaux, bactéries E. coli). 
Tableau 1 : Outils de collecte de données.

\begin{tabular}{lc}
\hline Outils / matériel et équipements & Utilisation \\
\hline Carte géographique de la commune & Connaissance géographique de la commune. \\
\hline Global Positionning System (GPS) & $\begin{array}{c}\text { Prise des coordonnées géographiques des puits, latrines, } \\
\text { puisards... }\end{array}$ \\
\hline Bouteilles teintées de capacité 2 litres & $\begin{array}{c}\text { Prélèvement des échantillons d'eau pour les analyses } \\
\text { chimiques }\end{array}$ \\
\hline $\begin{array}{l}\text { Flacon de prélèvement d'eau pour la } \\
\text { microbiologie }\end{array}$ & $\begin{array}{c}\text { Prélèvement des échantillons d'eau pour les analyses } \\
\text { microbiologiques }\end{array}$ \\
\hline $\begin{array}{l}\text { Glacière contenant des accumulateurs } \\
\text { de froid }\end{array}$ & $\begin{array}{c}\text { Conservation et transport des échantillons d'eau pour } \\
\text { des travaux en laboratoire }\end{array}$ \\
\hline pH-mètre, Multi paramètres & Mesure in-situ de pH et de la température de l'eau. \\
\hline Oxymètre & Mesure in-situ de l'oxygène dissous \\
\hline
\end{tabular}

\section{RESULTATS}

\section{Distance entre les puits et les latrines proches}

Les travaux de terrain ont permis de noter les distances qui séparent les puits échantillonnés des latrines les plus proches de ces puits. En matière d'hygiène et d'assainissement de base en République du Bénin, cette distance minimale doit être supérieure ou égale à 15 mètres. Et la latrine doit être en aval du puits. La Figure 2 présente les résultats des différentes mesures prises sur le terrain.

De l'analyse de cette Figure, il ressort que sur les 20 puits étudiés, 14 ont une distance inférieure à 15 mètres des latrines (distance recommandée par l'OMS). C'est le cas par exemple des puits $\mathrm{n}^{\circ} 2 ; 3 ; 4 ; 5 ; 6$; $7 ; 9 ; 10 ; 11 ; 12 ; 13 ; 15$ et 16 En effet, dans cette localité, les fosses de ces latrines sont étanchéifiées par du ciment. Mais cette étanchéité n'est pas efficace car parfois présente des fissures et de plus, la proximité de la nappe phréatique avec la surface du sol augmente le risque de transfert de contaminants fécaux de ces latrines vers les puits.

\section{Analyses physico-chimiques}

Les données du GPS (Global
Positionning System), ont permis de localiser les sites étudiés. La prise des coordonnées géographiques des infrastructures (puits, latrines, et autres ouvrages d'assainissements) sont représentées sur les Figures 3 et 4 .

Les différentes analyses effectuées ont donné les résultats suivants :

\section{Température}

La Figure 5 rend compte de la variation de la température de l'eau dans les puits. Les valeurs de la température des eaux prélevées en octobre 2015 sont comprises entre 24 et $28^{\circ} \mathrm{C}$. C'est en saison sèche que la plus forte valeur $\left(28{ }^{\circ} \mathrm{C}\right)$ a été enregistrée au niveau des puits $\mathrm{n}^{\circ} 2 ; 5 ; 19$ et 20 .

La Figure 6 présente la variation du $\mathrm{pH}$ de l'eau dans les puits échantillonnés entre octobre 2015 et janvier 2016. Les valeurs de $\mathrm{pH}$ obtenues au cours des deux périodes de cette étude: saison des pluies (mois d'octobre) et saison sèche (mois de janvier) sont comprises entre 6,6 et 7,8 pour l'ensemble des 20 puits. Ces valeurs de $\mathrm{pH}$ sont dans les limites de la norme nationale fixée pour la qualité de l'eau de consommation $(6<\mathrm{pH}<9)$.

Un pH compris entre 6 et 9 permet un développement à peu près correct de la faune et de la flore. Les organismes vivants sont très 
sensibles aux variations brutales même limitées du $\mathrm{pH}$.

\section{Oxygène dissous}

La concentration en oxygène dissous est un paramètre essentiel dans le maintien de la vie. Les variations des valeurs des concentrations en oxygène des eaux prélevées sont présentées dans la Figure 7.

\section{Les ions nitrates, nitrites, ammonium et phosphates}

Le nitrate, nitrite et ammonium sont des formes d'azote qui existent naturellement dans l'environnement. Les sources les plus courantes d'ions nitrates dans l'eau de puits sont : les engrais chimiques employés pour améliorer la croissance des cultures; les déchets d'origine animale; les sols contenant des composés d'azote provenant de matières organiques en décomposition et les installations d'assainissement (latrines) mal réalisées.

De même, les phosphates dans l'eau de puits ont une source naturelle : altération des roches; anthropiques : utilisation des engrais phosphatés, contamination par les déjections animales.

Les Figures 8, 9, 10 et 11 rendent compte de la variation des teneurs en nitrate, nitrite, ammonium et phosphate dans les échantillons d'eau prélevés au cours de la saison des pluies (mois d'octobre).

\section{Nitrates}

La concentration en nitrate varie entre 6 et $100 \mathrm{mg} / \mathrm{L}$. La valeur maximale permise pour une eau destinée à la consommation humaine selon le décret $n^{\circ} 2001-094$ du 20 février 2001 fixant les normes de qualité de l'eau potable en République du Bénin, est de $45 \mathrm{mg} / \mathrm{L}$.

\section{Nitrites}

La concentration en nitrite varie entre 0,02 et $5 \mathrm{mg} / \mathrm{L}$. Les taux de nitrites dans les puits $\mathrm{n}^{\circ} 1 ; 2 ; 3 ; 5 ; 9 ; 11 ; 13 ; 14 ; 15 ; 16$; $17 ; 19$ et 20 sont au-delà de la norme 0,1 $\mathrm{mg} / \mathrm{L}$ recommandée par la norme béninoise. Ce qui vient appuyer la mauvaise qualité de l'eau des puits révélée par les fortes valeurs de nitrates.

\section{Ammonium}

L'analyse de la variation du taux d'ammonium dans les puits échantillonnés montre que, la concentration en ammonium varie entre 0 et $40 \mathrm{mg} / \mathrm{L}$. Les valeurs guides en matière de potabilité de l'eau en République du Bénin recommande pour l'ammonium, des valeurs inférieures à 0,5 $\mathrm{mg} / \mathrm{L}$. Ainsi, les échantillons des puits 2, 4, 9 $11,12,13,14,16,17$ et 19 ne respectent pas cette norme.

10 : Valeurs des ions ammonium mesurées dans les échantillons d'eau des puits en octobre

\section{Phosphates}

La concentration en ions phosphates des échantillons d'eau de puits varie entre 0,75 et $60 \mathrm{mg} / \mathrm{L}$. La valeur maximale permise pour une eau destinée à la consommation humaine est de $5 \mathrm{mg} / \mathrm{L}$. Donc les échantillons $\mathrm{n}^{\circ} 3,11,13,14,15,16,17,19$ et 20 ne respectent pas cette norme et vient confirmée l'idée de pollution anthropique des eaux de puits.

\section{Analyses microbiologiques}

Les analyses microbiologiques ont concerné la recherche des coliformes totaux et des Escherichia colis (germes pathogènes), identifiés comme étant un indicateur de pollution d'origine fécale. Le dénombrement de ces germes a donné des valeurs qui sont consignées dans le Tableau 2. 


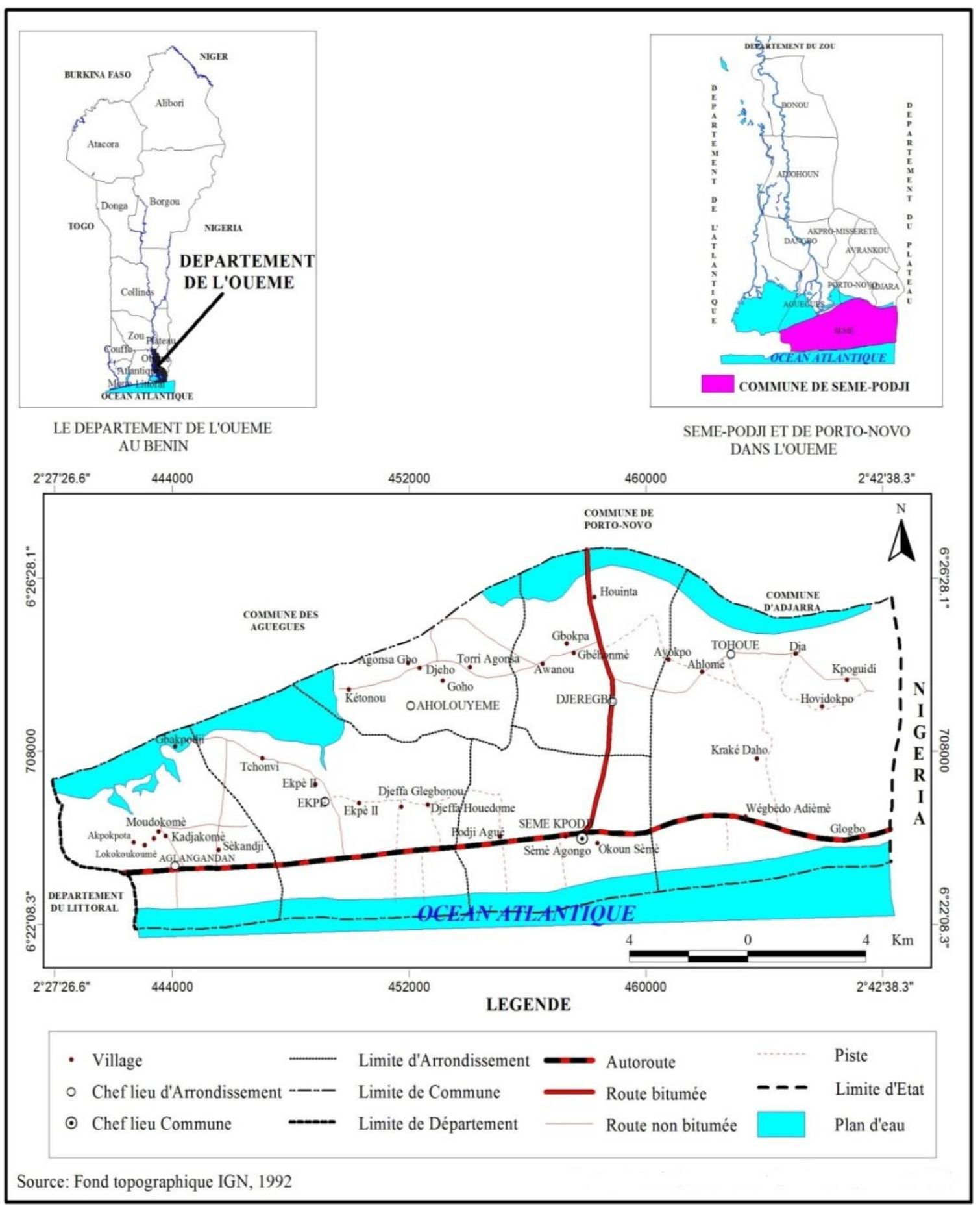

Figure 1 : Situation géographique de la commune de Sèmè-Podji. 


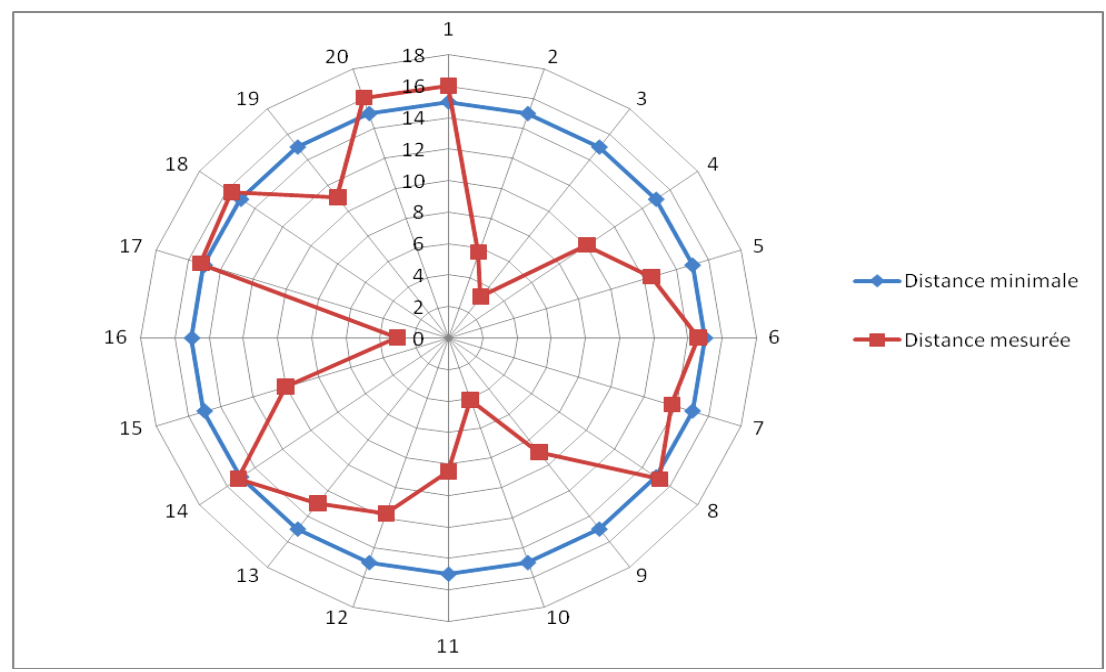

Figure 2 : Distance entre le puits et la latrine la plus proche.

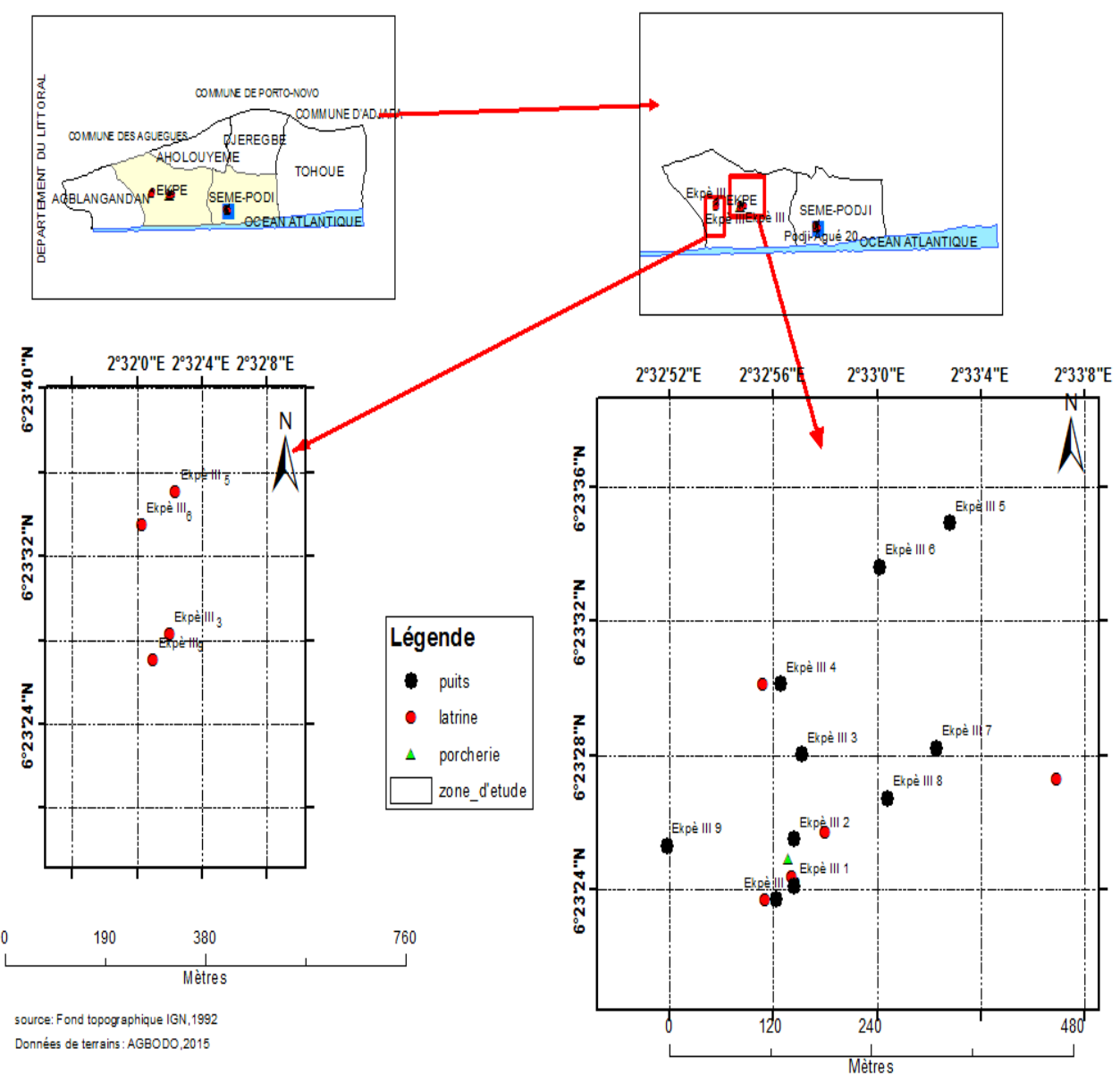

Figure 3 : Projection des ouvrages d'assainissement autonomes et points d'eaux étudiés à Ekpè. 


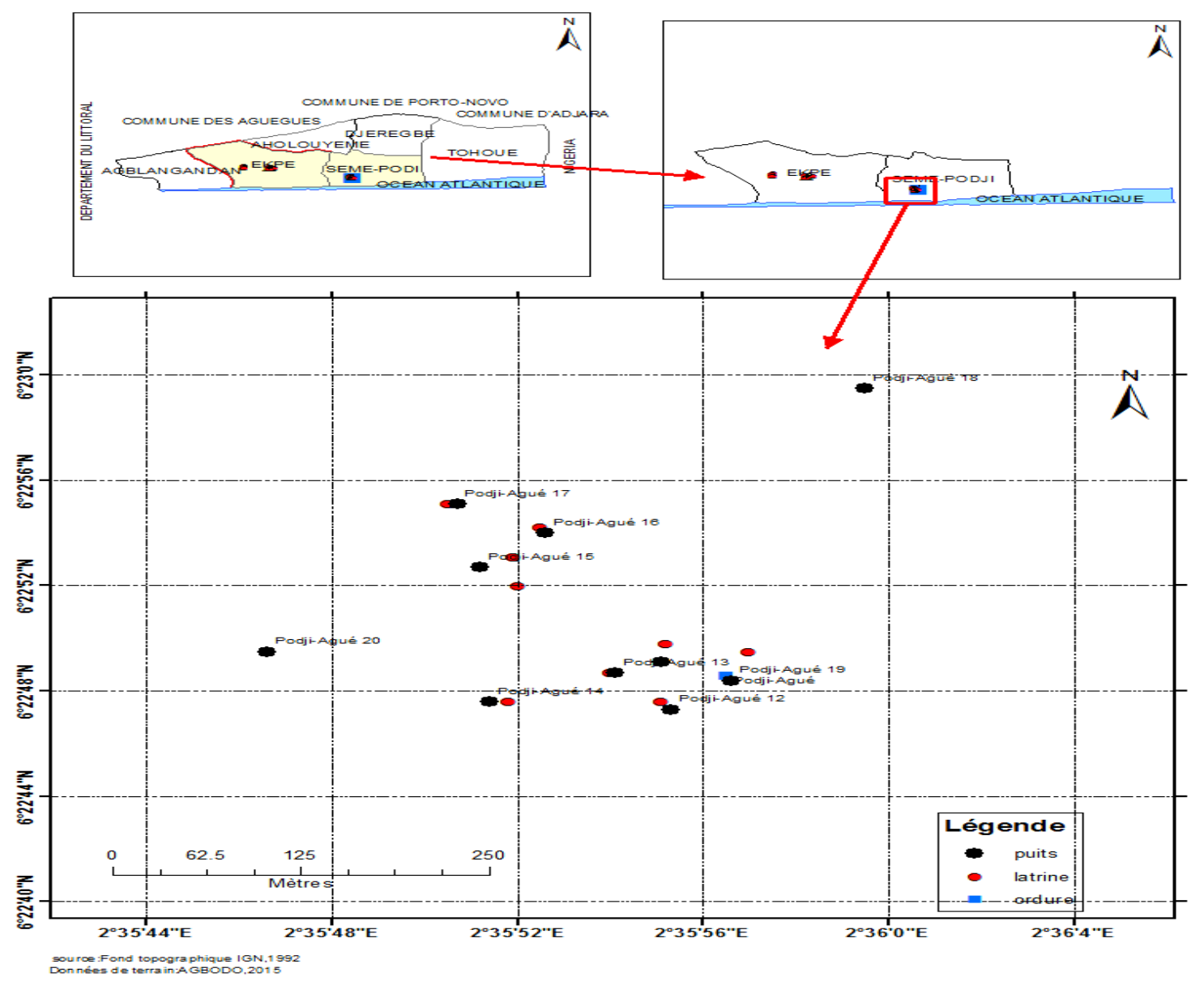

Figure 4 : Projection des ouvrages d'assainissement autonomes et points d'eaux étudiés à PodjiAgué.

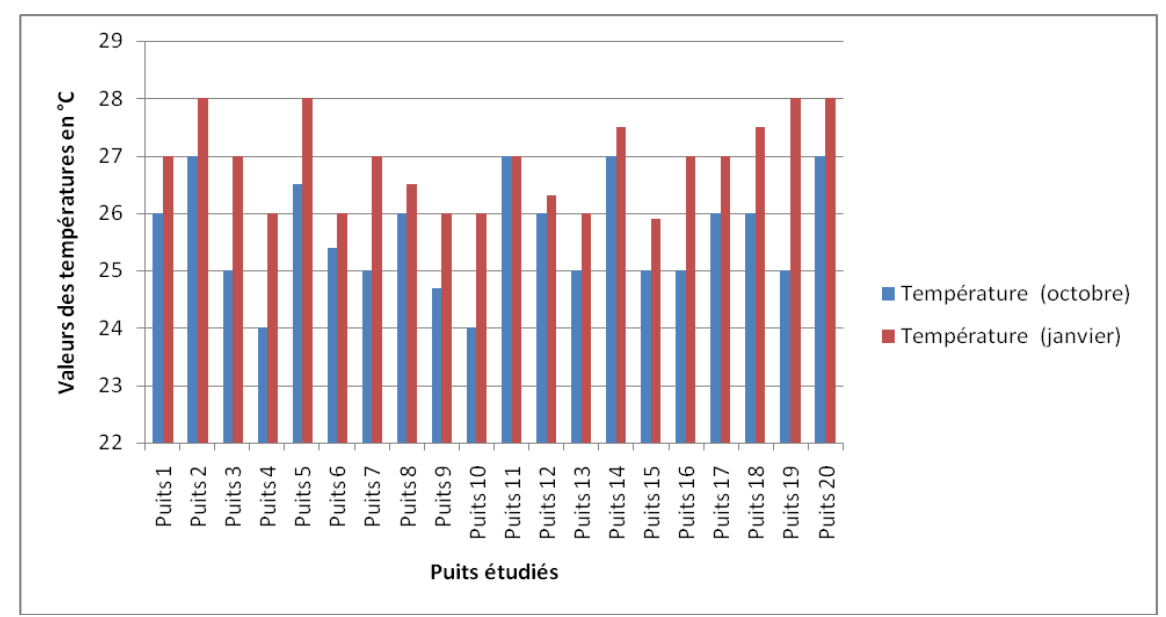

Figure 5 : Variation de la température des eaux de puits en octobre 2015 et en janvier 2016. 


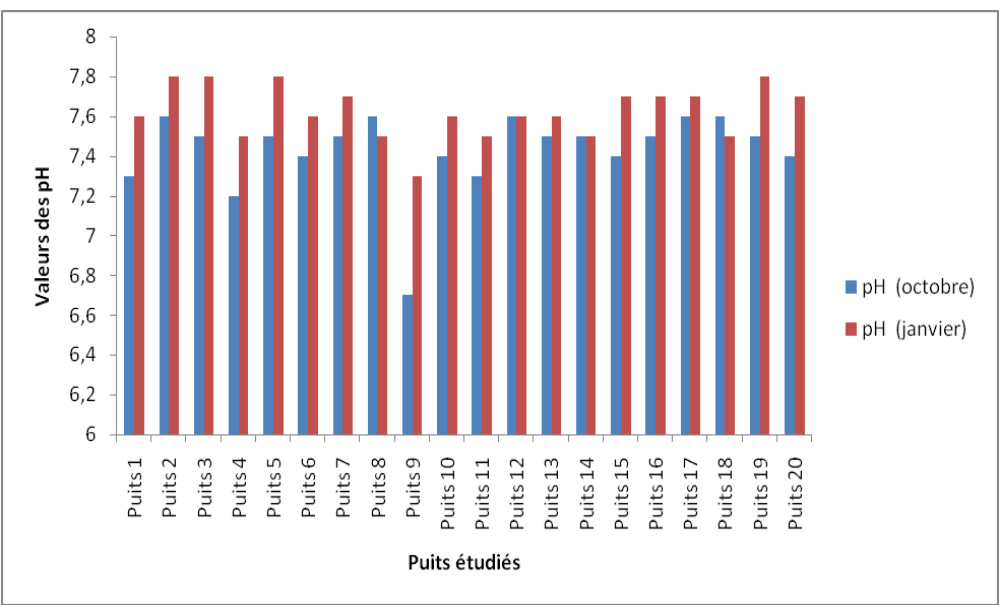

Figure 6 : Variation du pH des eaux de puits en octobre 2015 et en janvier 2016.

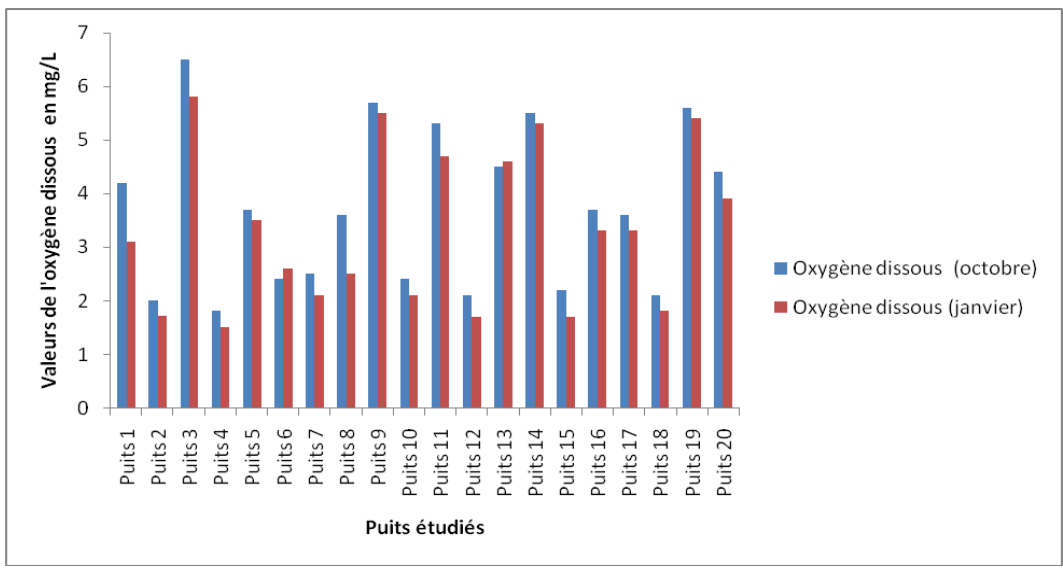

Figure 7 : Variation des taux d'oxygène des eaux de puits en octobre 2015 et en janvier 2016.

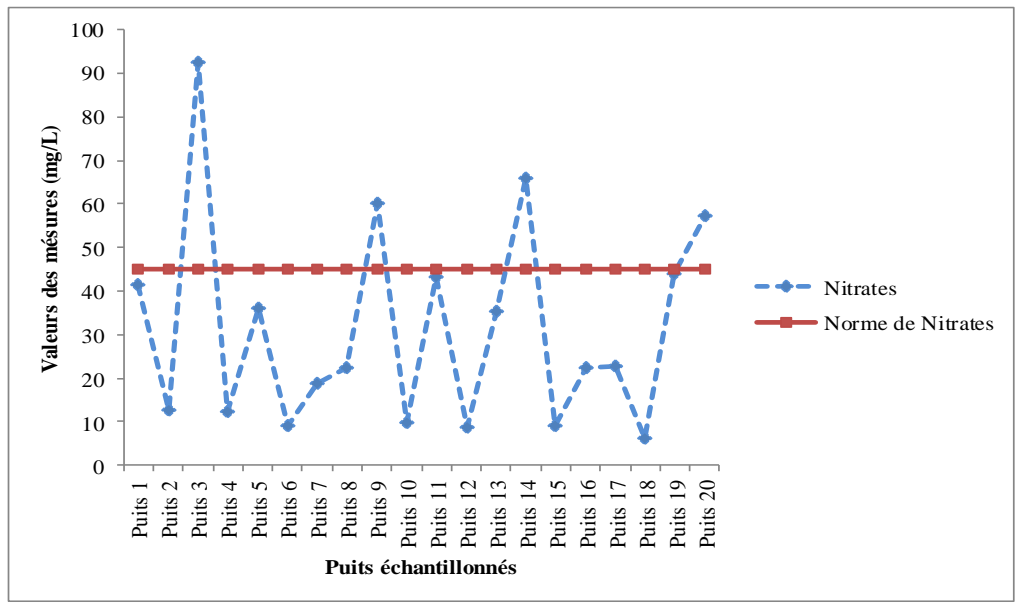

Figure 8: Valeurs des ions nitrates mesurées dans les échantillons d'eau des puits en octobre 2015. 


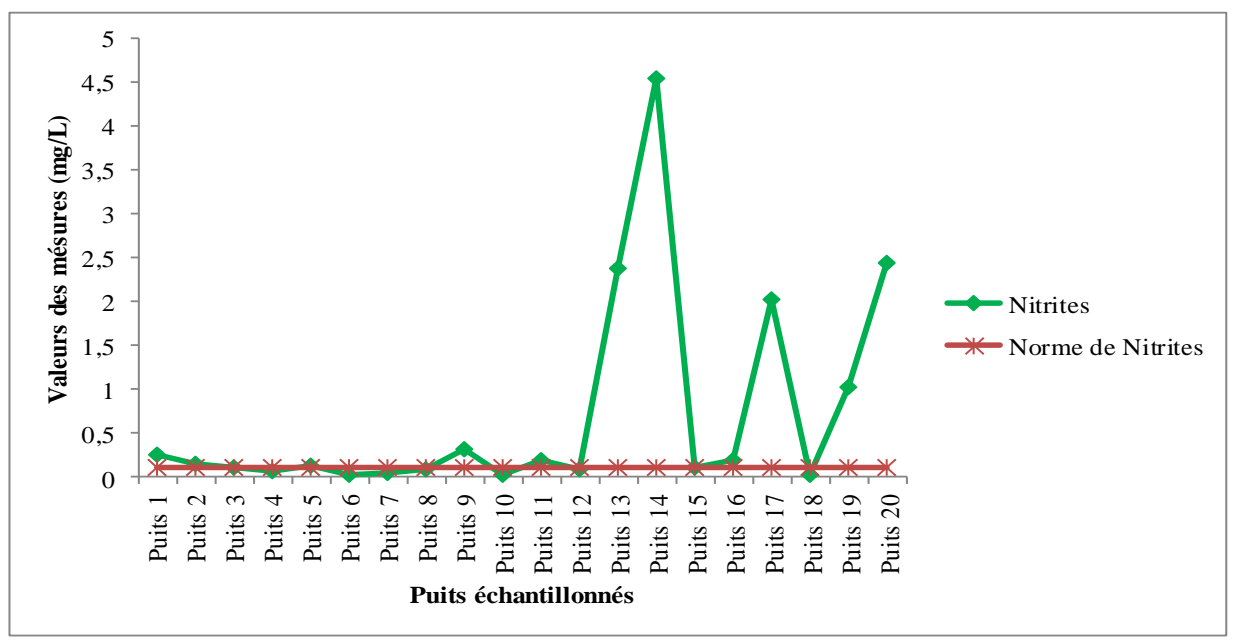

Figure 9 : Valeurs des ions nitrites mesurées dans les échantillons d'eau des puits en octobre 2015.

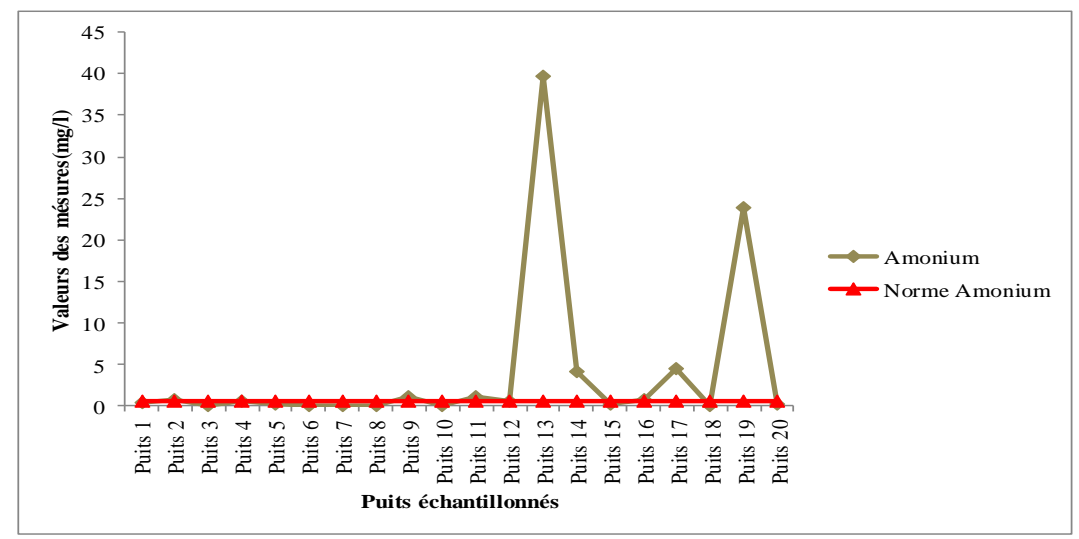

Figure 10 : Valeurs des ions ammonium mesurées dans les échantillons d'eau des puits en octobre.

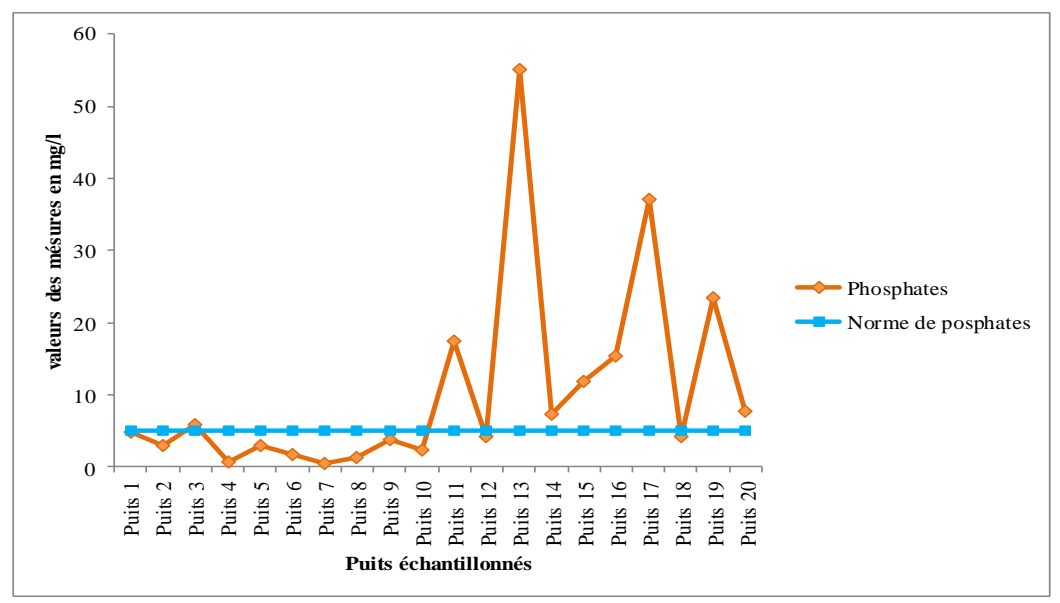

Figure 11 : Valeurs des ions phosphates mesurées dans les échantillons d'eau des puits en octobre 2015. 
Tableau 2 : Répartition des puits en fonction des germes identifiés.

\begin{tabular}{lccccc}
\hline $\begin{array}{l}\mathbf{N}^{\circ} \mathbf{d e} \\
\text { Puits }\end{array}$ & $\begin{array}{c}\text { Coliformes } \\
\text { Totaux }(\text { Rouge }) \\
\mathbf{a} \mathbf{3}{ }^{\circ} \mathbf{C}\end{array}$ & $\begin{array}{c}\text { E. Coli }(\text { Bleu }) \\
\text { à } \mathbf{3 7}{ }^{\circ} \mathbf{C}\end{array}$ & $\begin{array}{c}\mathbf{N}^{\circ} \text { de } \\
\text { Puits }\end{array}$ & $\begin{array}{c}\text { Coliformes Totaux } \\
\text { (Rouge) à } \mathbf{3 7}{ }^{\circ} \mathbf{C}\end{array}$ & $\begin{array}{c}\text { E. Coli (Bleu) à } \\
\mathbf{3 7}{ }^{\circ} \mathbf{C}\end{array}$ \\
\hline 1 & 2000 & 0 & 11 & 42400 & 0 \\
2 & 600 & 0 & 12 & 9000 & 0 \\
3 & 1100 & 0 & 13 & 21400 & 0 \\
4 & 200 & 0 & 14 & 1100 & 0 \\
5 & 4400 & 0 & 15 & 9100 & 0 \\
6 & 13100 & 0 & 16 & 1500 & 0 \\
7 & 7700 & 0 & 17 & 1200 & 0 \\
8 & 1200 & 0 & 18 & 1200 & 0 \\
\hline 10 & 700 & 0 & 19 & 9500 & 0 \\
\hline
\end{tabular}

\section{DISCUSSION}

La montée de la température de l'eau des puits en saison sèche pourrait se justifier par l'augmentation du rayonnement solaire pendant cette période de l'année. Une température élevée réduit la solubilité des gaz dans l'eau et en particulier les teneurs en oxygène. Ainsi, si la température de l'eau varie de 13 à $20{ }^{\circ} \mathrm{C}$, la concentration en oxygène chute de $13 \%$ (OMS, 2006). Les basses températures affectent la prolifération des germes microbiens car les réactions d'oxydation sont ralenties. Au contraire, une température plus élevée accélère ces réactions, mais entraîne par voie de conséquence une plus forte consommation d'oxygène dissous.

De l'analyse des variations de $\mathrm{pH}$ entre les deux périodes (période humide et période sèche), il ressort que le $\mathrm{pH}$ de l'eau en saison de pluie est plus bas qu'en saison sèche. Néanmoins, la quasi-totalité des valeurs fait état de ce que l'eau à un $\mathrm{pH}$ alcalin, donc plus favorable au développement d'une gamme plus large de germes microbiens.
Une analyse des valeurs au cours des deux périodes montre qu'en période sèche, relativement plus chaude, les taux d'oxygène dans les eaux des puits sont plus faibles que ceux de la période hivernale (mois d'octobre). Par ailleurs, on remarque que, les taux de la période sèche et ceux de la période des pluies sont presque identiques à quelques valeurs près. Les eaux des puits $\mathrm{n}^{\circ} 1 ; 3 ; 9 ; 11 ; 13$; $14 ; 16 ; 17 ; 19$ et 20 sont très riches en oxygène. Ces fortes valeurs d'oxygène sont favorables au développement des germes microbiens présents.

La concentration en nitrate varie entre 06 et $100 \mathrm{mg} / \mathrm{l}$. La valeur maximale permise pour une eau destinée à la consommation humaine par le décret n²001-094 du 20 février 2001 fixant les normes de qualité de l'eau potable en République du Bénin, est de $45 \mathrm{mg} / \mathrm{L}$. Ainsi, les échantillons des puits $\mathrm{n}^{\circ} 3$, 9, 14 et 20 ne respectent pas cette norme. Ils sont vis - à- vis de ce paramètre chimique, impropre à la consommation humaine. Quant à la concentration en nitrite, sa teneur varie entre 0,02 et $5 \mathrm{mg} / \mathrm{L}$. Les taux de nitrites dans 
les puits $\mathrm{n}^{\circ} 1 ; 2 ; 3 ; 5 ; 9 ; 11 ; 13 ; 14 ; 15$; $16 ; 17 ; 19$ et 20 sont au-delà de la norme 0,1 $\mathrm{mg} / \mathrm{L}$ recommandée par la norme béninoise. L'analyse de la variation du taux d'ammonium dans les puits échantillonnés montre que la concentration en ammonium varie entre 0 et $40 \mathrm{mg} / \mathrm{L}$. Les valeurs guides en matière de potabilité de l'eau en République du Bénin recommande pour l'ammonium, des valeurs inférieures à 0,5 $\mathrm{mg} / \mathrm{L}$. Ainsi, les échantillons des puits $\mathrm{n}^{\circ} 2,4$, $911,12,13,14,16,17$ et 19 ne respectent pas cette norme.

Pour ce qui concerne les ions phosphates, leur teneur varie entre 0,75 et 60 $\mathrm{mg} / \mathrm{L}$. La valeur maximale permise pour une eau destinée à la consommation humaine est de $5 \mathrm{mg} / \mathrm{L}$. Donc les échantillons $\mathrm{n}^{\circ} 3,11,13$, $14,15,16,17,19$ et 20 ne respectent pas cette norme et vient une fois encore confirmer l'idée de pollution anthropique des eaux de puits. Cette pollution qui peut être d'origine fécale ou animale ou liée à la décomposition des matières organiques enfouies dans le sol pourra être située avec les résultats d'analyses microbiologiques.

Ces résultats sont conformes à ceux obtenus par Edorh et al. (2007) qui ont noté que le non-respect de certaines règles d'hygiène, notamment la distance entre latrines et puits, la mise en place de dépotoirs d'ordures dans les zones inondables, met en cause la qualité des eaux de puits. Cette contamination est aussi due à la nature hydromorphe des sols, lesquels sont très sensibles à l'infiltration des eaux polluées du fait de leurs caractéristiques pédologiques (sols perméables, nappe phréatique affleurante). Ces eaux se retrouvent dans les puits par infiltration ou par déversement ; ceci confirme les observations faites par Agassounon (2012), qui avait noté que presque la totalité des puits des plaines inondables sont pollués par les infiltrations d'eaux usées.

A Hoteyi et al. (2014) ont déterminé une pollution azotée (concentration en nitrates variant de 75 à $97 \mathrm{mg} / \mathrm{l})$ des eaux de puits utilisées pour la production de l'eau en sachet dans la ville de Porto-Novo. De même, les eaux de cent dix (110) puits étudiées par Degbey et al. (2009) à Abomey-Calavi ont présenté une forte pollution en nitrates $(32,73 \%)$, en nitrites $(11,82 \%)$, en aluminium $(10 \%)$, en fer $(43,64 \%)$ total, en cadmium $(6,36 \%)$ et en mercure $(14,55 \%)$. Dans la même ville, les études de Hounsinou et al. (2015) sur les eaux de (20) puits ont donné des taux de conformité aux normes de l'OMS de $40 \%$ (pH), 60\% (conductivité électrique), 65\% (turbidité) et $100 \%$ (zinc). Dans la Commune de Pobè, les travaux de Lagnika et al. (2014) ont aussi identifié une pollution azotée se traduisant par des taux élevés en nitrates et en ammonium dans plus de 50\% des douze (12) puits étudiés.

\section{Conclusion}

Les analyses chimiques et bactériologiques sont les seuls moyens pour qualifier une eau de potable. L'eau, malgré ses caractéristiques organoleptiques acceptables (couleur, odeur, saveur), peut constituer un danger pour la santé humaine. C'est le cas des eaux analysées dans la Commune de SèmèPodji qui présentent une concentration très élevée en sels azotés notamment les nitrates et les nitrites. Elles contiennent également des microbes indicateurs de pollution d'origine animale et/ou fécale. Ces puits ne sont lavés que rarement ou mal lavés ce qui provoque souvent des maladies hydriques. Les latrines sont construites parfois non loin de ces puits. Il faut souligner ici que malgré le respect de la distance minimale de 15 mètres entre les puits et les latrines dans certains cas rares, l'eau est tout de même polluée. Il est donc souhaitable que les autorités locales se penchent sur cette situation pour éviter à l'avenir des problèmes d'épidémie de maladies hydriques dans cette localité du sud-Bénin.

\section{CONFLIT D'INTERETS}

Les auteurs attestent qu'il n'y a aucun conflit d'intérêts. 


\section{REMERCIEMENTS}

Les auteurs remercient l'Institut National de l'Eau de l'Université d'AbomeyCalavi pour sa contribution dans la réalisation de cet article.

\section{REFERENCES}

Agassounon Djikpo Tchibozo M, Ayi-Fanou L, Lozes E, Fadonougbo R, Anago GDJ, Agbangla C, Ahanhanzo C. 2012. Impacts sanitaires liés à l'usage des eaux de puits, à l'assainissement et à l'aménagement à Gbôdjê dans l'arrondissement de Godomey au Bénin. Int. J. Biol. Chem. Sci., 6(2): 592-602.

Ahoudi H, Gnandi K, Tanouayi G, OuroSama K. 2015. Caractérisation PhysicoChimique et Etat de pollution par les élements traces métalliques des eaux souterraines de Lomé (Sud Togo) : cas $\mathrm{du}$ quartier Agoe Zongo. Larhyss Journal, 24: 41-56.

Dovonou E. F. 2012. Diagnostic qualitatif et environnemental de l'aquifère superficiel du champ de captage intensif de Godomey au Bénin (Afrique de l'Ouest) : éléments pour un plan d'actions stratégiques de protection des ressources en eau souterraine exploitées. Doctorat ès Science en Hydrologie et Gestion Intégrée des Ressources en Eau. Université d'Abomey, Bénin, p.143

Dégbey C, Makoutodé M, Ouendo EM, De Brouwer C. $2009 . \quad$ Pollution physicochimique et microbiologique de l'eau des puits dans la Commune d'Abomey-Calavi au Bénin en 2009. Int. J. Biol. Chem. Sci., 4(6): 2257-2271.
Edorh A, Gnandi K, Elegbede Manou B, Enonhedo SF, Boko M. 2007. Qualité de l'eau des nappes et son impact sur la santé des populations de Kérou. Clim. Dével., 4: 27-37.

Hoteyi SMI, Gnimadi CC, Adjadji GV, Igoué AM, Mensah GA. 2014. Analyse des risques de consommation des eaux en sachet pour les populations dans ville de Porto-Novo au Sud-Bénin. Bulletin de la Recherche Agronomique du Bénin $(B R A B)$ Numéro spécial Agrobiodiversité et Santé publique, p. 8.

Hounsinou P, Mama D, Dovonou F. Alasane A. 2015. Seasonal evolution of the quality microbiological of the natural waters in the township of AbomeyCalavi (South Benin). British Journal of Earth Sciences Research, 3(1): 30-41.

INSAE. 2007. Analyse statistique. Ann. Insti. Nat. Stat. Ana. Eco., 23-35.

Lagnika M, Ibikounle M, Montcho J-PC, Wotto VD, Sakiti NG. 2014. Caractéristiques physico-chimique de l'eau de puits dans la commune de Pobè, Bénin. Journal of Applied Biosciences, 79: 6887-6897.

Organisation mondiale de la santé (OMS). 2006. Water and Sanitation Related Diseases fact, OMS. 